\title{
Crick's immodest ambitions
}

As scientists and others worldwide celebrate the 50th anniversary of a profound discovery, one of the discoverers persists with another challenging quest that, while still a work in progress, also deserves celebration.

W inning a Nobel Prize is a life-changing event. It instantly accords influence and status to its recipient in domains far outside the realm of the discipline being honoured. Some use this status to influence science policy. Many use it to attract donations or investment to their institutions or other worthy causes. More rarely, a laureate will apply the same principles that guided them to Nobel honours to continue their groundbreaking career in another scientific field. Francis Crick is one of the latter: decades after his discoveries about the root of life, DNA, he continues to investigate deep scientific questions by turning his talents to the root of consciousness, the brain.

Nature has not only had the privilege of publishing Crick's seminal contributions to our understanding of the molecular basis of life, but for the past two decades has also had the pleasure of publishing papers from him on various topics in cognitive neuroscience. Often in collaboration with leading neuroscientists, he has focused on the functional organization of the brain. For example, he has tackled the question of why we sleep, by considering the brain as a neural network whose organization may need to be periodically refreshed. He has described the impoverished status of studies in human neuroanatomy. And with Christof Koch he has written extensively about visual awareness and consciousness. In particular, Crick has championed the study of the neural correlates of consciousness: to use an example from his book The Astonishing Hypothesis (Simon \& Schuster, 1994), you perceive something to be red "if and only if certain neurons and/or molecules in your head behave in a certain way".

When Crick turned his attention to neuroscience, neurobiological investigations into consciousness were far from mainstream. Philosophers regarded consciousness as their exclusive domain, only occasionally ceding territory to neuropsychologists, who brought insights by studying patients such as those with blind-sight or split brains. Fundamental neuroscience was not seen as having much to offer, even by its own practitioners. The great nineteenth-century neurophysiologist Emil Du Bois-Reymond famously declared the scientific exploration of consciousness impossible ("ignoramus et ignorabimus"), and that view persists among some neuroscientists even today.

\section{Making connections}

But Crick has helped to bring consciousness and neuroscience together, emboldened by the confidence that led James Watson to observe mischievously that he had "never seen Francis Crick in a modest mood". Many great thinkers have turned to the human mind as the last great frontier of scientific endeavour. But few have done it with the rigorous attention to the neuroscience - from singleunit physiology to cognitive psychology - that must inform any biologically based theory of consciousness.

Having catalysed one scientific revolution by answering the correctly posed reductionist question, Crick approached consciousness through a similar search for the appropriate questions to ask. Rather than endlessly debating a precise definition of consciousness, Crick and his colleagues have focused on visual awareness as a phenomenon that is both central to the human experience and amenable to research with animal models. Rather than turn to vague ideas of 'emergent' brain properties, they have taken a first step to specificity, proposing that the activity of specific populations of neurons correlates with specific conscious phenomena.

Neuroscience still lacks the tools to accumulate data rapidly, so it is too early to assess the impact of Crick's specific proposals. Nonetheless, his questions are concrete enough to be taken as a challenge rather than a discouragement. It is not enough to know how a certain percentage of the neurons in a particular area respond during visual perception; we must identify the neurons involved, and know where they project and what neurons connect to them, before we can prove or disprove the idea that visual awareness is a function of higher areas of the cerebral cortex. Neuroscientists are constantly pushing the limits of the techniques available to them, of course, and Crick was not the first to point out that information of this kind is needed. But his powerful advocacy of the neural-correlates approach has helped to stimulate the community to develop new methods of answering the questions that he posed and of asking still more sophisticated ones.

\section{All in the mind}

More generally, neuroscience suffers from a shortage of strong theories. We would all agree that cracking the mysteries of the brain requires new hypotheses, but theorists are often undervalued. There seems to be simultaneously too much data to manage and yet too little data that can rigorously constrain a particular theory. It is too easy, many feel, to come up with a plausible neural explanation for a particular cognitive phenomenon, but too difficult to support or falsify it definitively. The framework for studying consciousness that Crick and his colleagues continue to articulate is one example of a guiding theoretical force that, once appropriate experimental tools become available, will not remain an unfalsifiable 'just so' story.

The insatiable public appetite for explanations of the mind is too often met with the intellectual equivalent of junk food. In contrast, Crick has brought the neuroscience of consciousness into the realm of the educated general public with The Astonishing Hypothesis. The challenge of bringing a unified theory of mind and brain to a population that largely believes the two to be separate entities requires both prominence and a clear voice.

The ideas on the neurobiological basis of consciousness that Crick has put forward over the years are not tightly bound into one consistent theory. They are, instead, explorations in a vast ocean of possibilities. They encourage neuroscientists to meet the challenge of this daunting topic. Like the question 'What is life?' - posed by an earlier Nobel laureate, Erwin Schrödinger, who was also unafraid to step outside the discipline in which his name was made - 'What is consciousness?' may be recast as more information is accumulated. If so, it will have made the transition from philosophy to science.

Crick carries forward the courage of his conviction that consciousness is worthy of serious scientific investigation by being both an insightful critic and an advocate of neuroscience. In doing so, he gives us grounds to hope, and indeed to expect, that the problem of consciousness is best placed within the scientific domain. 